\title{
TITLE:
}

\section{Hydrodynamics of driven granular gases}

$\operatorname{AUTHOR}(\mathrm{S})$ :

Hayakawa, $\mathrm{H}$

CITATION:

Hayakawa, H. Hydrodynamics of driven granular gases. Physical Review E 2003, 68(3): 031304.

ISSUE DATE:

2003-09

URL:

http://hdl.handle.net/2433/50477

RIGHT:

Copyright 2003 American Physical Society 
PHYSICAL REVIEW E 68, 031304 (2003)

\title{
Hydrodynamics of driven granular gases
}

\author{
Hisao Hayakawa \\ Department of Physics, Yoshida-South Campus, Kyoto University, Kyoto 606-8501, Japan
}

(Received 8 April 2003; published 12 September 2003)

\begin{abstract}
Hydrodynamic equations for granular gases driven by the Fokker-Planck operator are derived. Transport coefficients appeared in Navier-Stokes order change from the values of a free-cooling state to those of a steady state. The mismatch between the granular temperature and the constant temperature of the heat bath produces a nontrivial steady state.
\end{abstract}

DOI: 10.1103/PhysRevE.68.031304

PACS number(s): 81.05.Rm, 47.20.-k, 05.20.Dd

\section{INTRODUCTION}

The gas kinetic theory of elastic particles played important roles in history of statistical mechanics $[1,2]$. When there are inelastic interactions among particles, the behavior of collections of particles is completely different from that of elastic particles: There are no equilibrium states and any spatially homogeneous states are no longer stable. Such a collection of particles having inelastic interactions is called the granular gas, whose physical realization can be observed in rings of planets, small planets, suspended particles in fluidized beds, aerosols, and rapid granular flows, etc. [3].

A typical example of granular gases can be found in aerosols or suspensions, etc., in which the buoyancy is balanced with gravity $[4,5]$. In dense suspensions the hydrodynamic interaction among particles are important [6], but effects of the air may be regarded as a thermostat driven by the Langevin forces in dilute suspensions. Most researches for granular gases are interested in undriven systems which are difficult to be achieved in actual experiences. We believe that more systematic studies for driven granular gases are required. Montanero and Santos analyzed statistical properties of the homogeneous state and hydrodynamics of granular gases, the white noise thermostat, and the Gaussian thermostat $[7,8]$. Carrillo et al. [9] and Pagnani et al. [10] analyzed granular gases in driven systems by the Langevin force which includes both the white noise and the friction force in proportion to the velocity of particles, but they are not interested in hydrodynamics of such a granular fluid. Since we believe that the model driven by such a thermostat, we call the Langevin thermostat, can describe physical situations of dilute suspensions, we need to clarify the properties of the hydrodynamics of such a system. The main purpose of this paper is to investigate the effects of Langevin thermostat for transport coefficients

The organization of this paper is as follows. In the following section, we introduce our model and the framework of the Chapman-Enskog method for the analysis of granular gases. In Sec. III, we calculate the valuables such as the granular temperature and the fourth cumulant in a homogeneous state. In Sec. IV, we obtain the transport coefficients such as the viscosity and the heat conductivity. In Sec. V, we discuss and summarize our results.

\section{FRAMEWORK}

We consider rarefied gases of smooth identical particles with the mass $m$, the velocity $\mathbf{v}$, and the diameter $\sigma$. The distribution function $f(\mathbf{r}, \mathbf{v}, t)$ in our system obeys the heated inelastic Boltzmann equation

$$
\left(\partial_{t}+\mathbf{v} \cdot \boldsymbol{\nabla}\right) f=J[f, f]+L_{F P} f,
$$

where $J[f, f]$ represents the collisional integral given by $[11,12]$

$$
\begin{aligned}
J[f, h]= & \sigma^{d-1} \int d \hat{\boldsymbol{\sigma}} \int d \mathbf{v}_{1} \Theta(\mathbf{g} \cdot \hat{\boldsymbol{\sigma}}) \mathbf{g} \cdot \hat{\boldsymbol{\sigma}}\left(e^{-2} b^{-1}-1\right) \\
& \times f(\mathbf{r}, \mathbf{v}, t) h\left(\mathbf{r}, \mathbf{v}_{1}, t\right),
\end{aligned}
$$

where $\Theta(x)$ is the Heaviside function, $\mathbf{g}=\mathbf{v}-\mathbf{v}_{1}$, and $\hat{\boldsymbol{\sigma}}$ is the unit vector along the line connecting centers of mass of contacting particles. The operator $b^{-1}$ is the inverse of the collisional operator $b$ which are defined as

$$
\begin{aligned}
& b \mathbf{g}=\mathbf{g}-(1+e)(\mathbf{g} \cdot \hat{\boldsymbol{\sigma}}) \hat{\boldsymbol{\sigma}}, \\
& b^{-1} \mathbf{g}=\mathbf{g}-\frac{1+e}{e}(\mathbf{g} \cdot \hat{\boldsymbol{\sigma}}) \hat{\boldsymbol{\sigma}},
\end{aligned}
$$

where $e$ is the coefficient of restitution which is ranged 0 $<e \leqslant 1$. Here we assume that $e$ is a constant for the simplification of our argument, though the actual coefficient of restitution depends on the impact velocity [13-15]. The effects of the impact velocity dependence of $e$ to macroscopic hydrodynamics can be seen in Ref. [16]. It should be noted that the oblique impacts have important contributions in actual inelastic collisions $[17,18]$. We, however, assume that the effects of inelastic oblique collision can be neglected, which may be justified when particles are smooth hard-core particles.

The Fokker-Planck operator $L_{F P}$ in Eq. (1) represents the driven force coming from the Langevin force as

$$
L_{F P}=\gamma_{0} \frac{\partial}{\partial \mathbf{v}} \cdot\left[\mathbf{V}+\frac{T_{B}}{m} \frac{\partial}{\partial \mathbf{v}}\right]
$$

where the first term and the second term represent the frictional force and the thermal activation, respectively. In general, the temperature of the heat bath $T_{B}$ is different from granular temperature $T$. Here, we assume that $T_{B}$ is a constant throughout the argument in this paper. This assumption is justified when the influence of granular particles to the heat bath is negligible. If there is no contribution from the 
collisional integral, the distribution function is relaxed to an equilibrium state as $f \rightarrow f_{e q} \propto \exp \left[-m V^{2} / 2 T_{B}\right]$. We note that the Fokker-Planck operator (5) is different from white noise thermostat $L_{\text {white }}=\left(\gamma_{0} T / m\right)\left(\partial^{2} / \partial v^{2}\right)$ even when we neglect the contribution the friction term. Actually, our $L_{F P}$ is reduced to $\gamma_{0} T_{B} / m\left(\partial^{2} / \partial v^{2}\right)$ in such a situation with a constant temperature $T_{B}$. We also note that the frictional force in the first term of Eq. (5) has the inverse sign of the Gaussian thermostat [7] $L_{\text {Gauss }}=-\gamma_{0}(\partial / \partial \mathbf{v}) \mathbf{V}$ which is also used in simulation of molecular hydrodynamics [19].

Hydrodynamic variables which characterize the macroscopic behavior of the gas are the number density, the velocity field, and the granular temperature defined by

$$
\begin{gathered}
n(\mathbf{r}, t)=\int d \mathbf{v} f(\mathbf{r}, \mathbf{v}, t), \\
n(\mathbf{r}, t) \mathbf{u}(\mathbf{r}, t)=\int d \mathbf{v} \mathbf{v} f(\mathbf{r}, \mathbf{v}, t), \\
\frac{d}{2} n(\mathbf{r}, t) T(\mathbf{r}, t)=\int d \mathbf{v} \frac{1}{2} m \mathbf{V}^{2} f(\mathbf{r}, \mathbf{v}, t),
\end{gathered}
$$

where $\mathbf{V} \equiv \mathbf{v}-\mathbf{u}$. The integral of the collisional invariance multiplied by $J[f, f]$ over $\mathbf{V}$ is zero. Since the loss of kinetic energy in each collision is given by

$$
\Delta E=-\frac{1-e^{2}}{4} m(\mathbf{g} \cdot \hat{\boldsymbol{\sigma}})^{2},
$$

the following relation holds:

$$
\int d \mathbf{v} \frac{1}{2} m V^{2} J(f, f)=-\frac{n d}{2} T \zeta[f, f] .
$$

Here, the cooling rate $\zeta$ in Eq. (10) can be evaluated approximately [12]

$$
\zeta \simeq \zeta^{(0)}=\frac{1-e^{2}}{4 d} \nu_{0}(d+2)\left(1+\frac{3}{16} a_{2}\right),
$$

where $\nu_{0}=\pi^{-1 / 2} n \sigma^{d-1}(T / m)^{1 / 2} 4 \Omega_{d} /(d+2)$, with $\Omega_{d}$ $=2 \pi^{d / 2} / \Gamma(d / 2) . a_{2}$ is the fourth cumulant defined by

$$
\begin{gathered}
a_{2} \equiv \frac{d}{d+2} \frac{\left\langle V^{4}\right\rangle}{\left\langle V^{2}\right\rangle^{2}}-1, \\
\left\langle V^{k}\right\rangle \equiv \frac{1}{n} \int d \mathbf{V} V^{k} f(\mathbf{V}, t),
\end{gathered}
$$

which will be determined later.

The balance equations for hydrodynamic variables are

$$
\begin{gathered}
D_{t} n+n \boldsymbol{\nabla} \cdot \mathbf{u}=0, \\
D_{t} u_{i}+(m n)^{-1} \nabla_{j} P_{i j}=0, \\
D_{t} T+\frac{2}{d n}\left(P_{i j} \nabla_{j} u_{i}+\nabla \cdot \mathbf{q}\right)+T \zeta=2 \gamma_{0}\left(T_{B}-T\right),
\end{gathered}
$$

where $D_{t}=\partial_{t}+\mathbf{u} \cdot \boldsymbol{\nabla}$. The pressure tensor $P_{i j}$ and the heat flux $\mathbf{q}$ are, respectively, defined by

$$
\begin{gathered}
P_{i j}=m \int d \mathbf{v} V_{i} V_{j} f(\mathbf{r}, \mathbf{v}, t), \\
\mathbf{q}=\frac{m}{2} \int d \mathbf{v} V^{2} \mathbf{V} f(\mathbf{r}, \mathbf{v}, t) .
\end{gathered}
$$

We adopt the Chapman-Enskog method [2], where space and time dependences appear through hydrodynamic variables. The expansion parameter is regarded as the magnitude of spatial inhomogeneity. Thus, we expand $f$ around the homogeneous solution $f^{(0)}$ as $f=f^{(0)}+\varepsilon f^{(1)}+\varepsilon^{2} f^{(2)}+\cdots$, the time derivative is also expanded as $\partial_{t}=\partial_{t}{ }^{(0)}+\varepsilon \partial_{t}{ }^{(1)}+\cdots$. Here we apply the Chapman-Enskog method for dilute granular gases developed by Brey et al. [20,21] and Santos [8] to driven systems. To remove the ambiguity of the distribution function, we impose the solubility conditions in which hydrodynamic variables are unchanged from the evaluation by $f^{(0)}(\mathbf{v}, t)$.

\section{HOMOGENEOUS STATES}

As the first step of Chapman-Enskog method, we need also to obtain the homogeneous solution of the inelastic Boltzmann equation. We usually assume the scaling form

$$
f(\mathbf{v}, t)=n v_{0}(t)^{-d} \widetilde{f}(\mathbf{c}, \tau), \quad \mathbf{c}=\mathbf{V} / v_{0}(t),
$$

with $d \tau=\omega_{E} d t$ and $v_{0}(t)=\sqrt{2 T / m}$. The fourth cumulant introduced in Eq. (12) for the scaling function is related to $\left\langle c^{4}\right\rangle \equiv \int d \mathbf{c} c^{4} \tilde{f}(\mathbf{c})$ as $\left\langle c^{4}\right\rangle=d(d+2)\left(a_{2}+1\right) / 4$. Here $\omega_{E}$ is Enskog's collision frequency, given by

$$
\omega_{E}=\frac{d+2}{4} \nu_{0}=\sqrt{\frac{2}{\pi}} \Omega_{d} n \sigma^{d-1} v_{0} .
$$

For the calculation we need to obtain

$$
\mu_{k} \equiv-\int d \mathbf{c} c^{k} J[\tilde{f}, \tilde{f}] .
$$

The cumulants and $\mu_{k}$ can be evaluated by an approximate expansion of Sonine polynomials [12]. For example, $\mu_{2}$ is evaluated as

$$
\mu_{2} \simeq \frac{1}{2}\left(1-e^{2}\right) \frac{\Omega_{d}}{\sqrt{2 \pi}}\left(1+\frac{3}{16} a_{2}\right) .
$$

$\mu_{4}$ is estimated as [12]

$$
\mu_{4} \simeq \sqrt{\frac{2}{\pi}} \Omega_{d}\left(A_{1}+a_{2}^{H} A_{2}\right),
$$

with

$$
A_{1}=\frac{1-e^{2}}{4}\left(d+\frac{3}{2}+e^{2}\right) \text {, }
$$




$$
A_{2}=\frac{3}{128}\left(1-e^{2}\right)\left(10 d+39+10 e^{2}\right)+\frac{1}{4}(1+e)(d-1) .
$$

It should be noted that these evaluations are based on two approximations: (i) the truncation of the first Sonine expansion and (ii) the linearization of $a_{2}$. If we adopt the first assumption, the linearization of $a_{2}$ gives a nice evaluation [22]. We also indicate that the direct comparison of the transport coefficients obtained by the Monte Carlo simulation and the linearized approximation gives good agreement in freecooling states [21]. However, nobody knows how to converge the Sonine expansion for driven granular gases. Thus, we may need to check the convergence of the Sonine expansion as in the case of elastic particles [23].

Now, let us discuss the time evolution of temperature field. Eq. (16) becomes

$$
\partial_{t}^{(0)} \theta=2 \gamma_{0}-\left(2 \gamma_{0}+\zeta\right) \theta
$$

in the homogeneous state with the Langevin thermostat, where $\theta \equiv T / T_{B}$. Assuming $\theta=\theta^{(0)}+a_{2} \theta^{(1)}+O\left(a_{2}^{2}\right)$, Eq. (25) is reduced to

$$
\partial_{\tau} \theta^{(0)}=2\left\{\hat{\gamma}-(\hat{\gamma}+\hat{\zeta}) \theta^{(0)}\right\}
$$

in the lowest order, where $\hat{\gamma}=\gamma_{0} / \omega_{E}$ and $\hat{\zeta}=\left(1-e^{2}\right) / 2 d$. This equation has the solution

$$
\theta^{(0)}=\theta_{\infty}+\left(\theta^{(0)}(0)-\theta_{\infty}\right) e^{-2(\hat{\gamma}+\hat{\zeta}) \tau}
$$

with $\theta_{\infty} \equiv \hat{\gamma} /(\hat{\gamma}+\hat{\zeta})$

In the scaling limit, the inelastic Boltzmann equation (1) is reduced to

$$
\begin{aligned}
\frac{\Omega_{d}}{\sqrt{2 \pi}} \partial_{\tau} \widetilde{f}(\mathbf{c}, \tau)= & \widetilde{J}[\widetilde{f}, \widetilde{f}]+\left(\frac{\hat{\gamma}}{\theta}-\frac{\mu_{2}}{d}\right) \frac{\partial}{\partial \mathbf{c}} \cdot[\mathbf{c} \widetilde{f}(\mathbf{c}, \tau)] \\
& +\frac{\hat{\gamma}}{2 \theta} \frac{\partial^{2}}{\partial c^{2}} \widetilde{f}(\mathbf{c}, \tau),
\end{aligned}
$$

where $J[f, f]=n^{2}\left(\sigma / v_{0}(t)\right)^{d-1} \widetilde{J}[\widetilde{f}, \widetilde{f}]$. From the equation for $\left\langle c^{4}\right\rangle=d(d+2)\left(a_{2}+1\right) / 4$ we obtain the equation of the lowest order of $a_{2}$ :

$$
\begin{aligned}
\partial_{\tau} a_{2} & =4 \hat{\zeta}-\hat{A}_{1}+a_{2}\left[\frac{19}{4} \hat{\zeta}-4 \frac{\hat{\gamma}}{\theta}-\hat{A}_{2}\right]+\frac{3}{4} \hat{\zeta} a_{2}^{2} \\
& \simeq 4 \hat{\zeta}-\hat{A}_{1}+a_{2}\left[\frac{19}{4} \hat{\zeta}-4 \frac{\hat{\gamma}}{\theta^{(0)}}-\hat{A}_{2}\right],
\end{aligned}
$$

with $\hat{A}_{1}=8 A_{1} / d(d+2)$ and $\hat{A}_{2}=8 A_{2} / d(d+2)$. The solution of Eq. (30) is given by

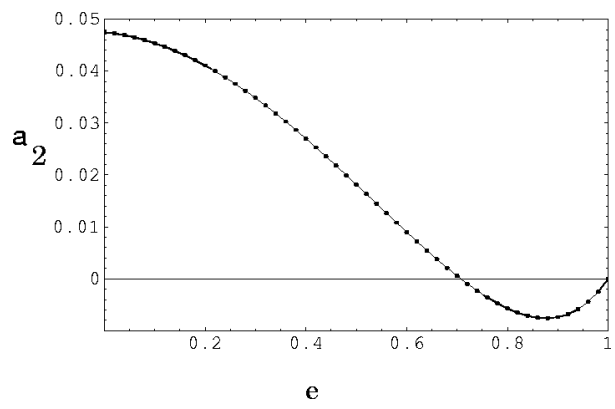

FIG. 1. Steady $a_{2}$ as a function of $e$ for $d=3$ and $\hat{\gamma}=0.1$. The solid line and the solid circles represent the result of linearized approximation and the exact one, respectively.

$$
\begin{aligned}
a_{2}(\tau) \simeq & a_{2}^{\infty}+\left[a_{2}(0)-a_{2}^{\infty}\right] \times \exp \left[\left(\frac{19}{4} \hat{\zeta}-\hat{A}_{2}\right) \tau\right. \\
& \left.-4 \hat{\gamma} \int_{0}^{\tau} \frac{d \tau^{\prime}}{\theta^{(0)}\left(\tau^{\prime}\right)}\right],
\end{aligned}
$$

where

$$
a_{2}^{\infty}=\frac{\hat{A}_{1}-4 \hat{\zeta}}{\frac{19}{4} \hat{\zeta}-4(\hat{\gamma}+\hat{\zeta})-\hat{A}_{2}}
$$

Although we present the result of linearization of $a_{2}$, it is possible to obtain the exact steady values of $\theta$ and $a_{2}$ for Eqs. (25) and (29). The result is presented in the Appendix, but the differences between the exact values and the result from linearized approximation are invisible (Fig. 1). We also compare $a_{2}$ in Eq. (31) and $\theta$ in Eq. (27) with the numerical solution of Eqs. (29) and (25) to confirm the validity of the linearization of $a_{2}$.

\section{THE DETERMINATION OF THE TRANSPORT COEFFICIENTS}

Here, we explicitly obtain the result of the transport coefficients for heated granular gases. The solution $f^{(0)}$ is isotropic so that the zeroth-order pressure and the heat flux are given by

$$
P_{i j}^{(0)}=p \delta_{i j}, \quad \mathbf{q}^{(0)}=\mathbf{0},
$$

where $p=n T$ is the hydrostatic pressure.

The first-order equation of the Boltzmann equation becomes

$$
\begin{aligned}
\left(\partial_{t}{ }^{(0)}+L-L_{F P}\right) f^{(1)} & =-\left(\partial_{t}{ }^{(1)}+\mathbf{v} \cdot \boldsymbol{\nabla}\right) f^{(0)} \\
& =-\left(D_{t}^{(1)}+\mathbf{V} \cdot \boldsymbol{\nabla}\right) f^{(0)},
\end{aligned}
$$

with $D_{t}^{(1)}=\partial_{t}^{(1)}+\mathbf{u} \cdot \boldsymbol{\nabla}$. Here the linear operator $L$ in Eq. (34) is defined by

$$
L f^{(1)}=-J\left[f^{(0)}, f^{(1)}\right]-J\left[f^{(1)}, f^{(0)}\right] .
$$


Multiplying both sides of Eq. (34) by $m V_{i} V_{j}$ and integrating over $\mathbf{V}$, we obtain

$$
\left(\partial_{t}^{(0)}+\nu\right) P_{i j}{ }^{(1)}+\Pi_{i j}{ }^{(1)}=-p \Delta_{i j k l} \nabla_{k} u_{l},
$$

where

$$
\begin{gathered}
\Pi_{i j}{ }^{(1)} \equiv-m \int d \mathbf{V} V_{i} V_{j} L_{F P} f^{(1)}, \\
\Delta_{i j k l} \equiv \delta_{i k} \delta_{j l}+\delta_{i l} \delta_{j k}-\frac{2}{d} \delta_{i j} \delta_{k l} .
\end{gathered}
$$

The solution of Eq. (36) can be written as

$$
P_{i j}^{(1)}=-\eta \Delta_{i j k l} \boldsymbol{\nabla}_{k} u_{l},
$$

where $\eta$ is the viscosity.

It is possible to obtain $\nu$ in Eq. (36) through the relation

$$
m \int d \mathbf{V} V_{i} V_{j} L f^{(1)}(\mathbf{V})=\nu P_{i j}{ }^{(1)} .
$$

The evaluation of $\nu$ is independent of the existence of the thermostat. We have evaluated $\nu$ as [12]

$$
\nu_{\eta}^{*} \equiv \frac{\nu}{\nu_{0}} \simeq \frac{3}{4 d}\left(1-e+\frac{2}{3} d\right)(1+e)\left(1-\frac{1}{32} a_{2}\right) .
$$

With the aid of

$$
\partial_{t}^{(0)} P_{i j}^{(1)}=\left(\frac{\gamma_{0}}{\theta}-\gamma_{0}-\frac{\zeta}{2}\right) P_{i j}^{(1)},
$$

Eqs. (36)-(42) lead to

$$
\eta^{*} \equiv \frac{\eta}{\eta_{0}}=\frac{2 \gamma^{*}+1}{\gamma^{*}\left(1+\theta^{-1}\right)+\nu_{\eta}^{*}-\zeta^{*} / 2},
$$

where $\gamma^{*}=\gamma_{0} / \nu_{0}, \nu_{\eta}^{*}=\nu / \nu_{0}$, and $\zeta^{*}=\zeta / \nu_{0} \cdot \eta_{0}(\hat{\gamma})$ is the viscosity for $e=1$, which is different from the value $\eta_{e}$ of the elastic gas as $\eta_{e}=\eta_{0}\left(2 \hat{\gamma}^{*}+1\right)$. We note $\eta_{e}$ $=5 \sqrt{\pi m T} /\left(16 \sqrt{\pi} \sigma^{2}\right)$ for $d=3$ in the lowest-order Sonine approximation [2]. The steady value of $\eta^{*}$ is obtained when we substitute $\theta^{\infty}$ and $a_{2}^{\infty}$ into Eq. (43).

Let us consider the heat flux. Multiplying both sides of Eq. (34) by $m V^{2} \mathbf{V} / 2$ and integrating over $\mathbf{V}$ we obtain

$$
\begin{aligned}
\left(\partial_{t}^{(0)}+\nu^{\prime}\right) \mathbf{q}^{(1)}+\mathbf{Q}^{(1)}= & -\frac{d+2}{2}\left(1+2 a_{2}\right) \frac{p}{m} \boldsymbol{\nabla} T \\
& -\frac{d+2}{2} a_{2} \frac{T^{2}}{m} \nabla n,
\end{aligned}
$$

where

$$
\mathbf{Q}^{(1)}=-\frac{m}{2} \int d \mathbf{V} V^{2} \mathbf{V} L_{F P} f^{(1)} .
$$

Here, $\nu^{\prime}$ has already been calculated as [21]

$$
\begin{aligned}
\nu_{\kappa}^{*} \equiv & \frac{\nu^{\prime}}{\nu_{0}} \simeq \frac{1+e}{d}\left[\frac{d-1}{2}+\frac{3}{16}(d+8)(1-e)\right. \\
& \left.+\frac{4+5 d-3(4-d) e}{512} a_{2}\right] .
\end{aligned}
$$

The heat flux is described by

$$
\mathbf{q}^{(1)}=-\kappa \boldsymbol{\nabla} T-\mu \boldsymbol{\nabla} n,
$$

where $\eta$ and $\kappa$ are the shear viscosity and the thermal conductivity, respectively. The other transport coefficient $\mu$ appears only in granular gases. Through the substitution of Eqs. (47) and (46) with the result of $\mathbf{Q}^{(1)}$ into Eq. (44) we can obtain $\kappa$ and $\mu$.

From the scaling form (19) the following relations should be satisfied:

$$
\begin{aligned}
\partial_{t}^{(0)} \mathbf{q}^{(1)}= & \left(2 \zeta+3 \gamma_{0}-\gamma_{0} / \theta\right) \kappa \boldsymbol{\nabla} T \\
& +\left\{\mu\left[\frac{3}{2} \zeta+3 \gamma_{0}\left(1-\theta^{-1}\right)\right]+\frac{\kappa \zeta T}{n}\right\} \boldsymbol{\nabla} n .
\end{aligned}
$$

On the other hand, $\Pi^{(1)}$ and $\mathbf{Q}^{(1)}$ have the relations

$$
\Pi_{i j}^{(1)}=2 \gamma_{0} P_{i j}^{(1)}, \quad \mathbf{Q}^{(1)}=3 \gamma_{0} \mathbf{q}^{(1)} .
$$

Thus, $\kappa$ and $\mu$ can be obtained from Eqs. (48), (49), and (44) as

$$
\kappa=\frac{d+2}{2} \frac{1+2 a_{2}}{\nu^{\prime}-2 \zeta+\gamma_{0} / \theta} \frac{n T}{m},
$$

$$
\mu=\frac{\frac{\kappa \zeta T}{n}+\frac{d+2}{2} a_{2} \frac{T^{2}}{m}}{\nu^{\prime}-\frac{3}{2} \zeta+3 \gamma_{0} / \theta} .
$$

Thus, $\kappa^{*}=\kappa / \kappa_{0}$ which is the heat conductivity normalized by its value of $e=1, \kappa_{0}(\hat{\gamma})$, is given by

$$
\begin{gathered}
\kappa^{*}=\left(\frac{d-1}{d}+\gamma^{*}\right) \frac{1+2 a_{2}}{\nu_{\lambda}^{*}-2 \zeta^{*}+\gamma^{*} / \theta}, \\
\mu^{*}=\frac{\kappa^{*}}{1+2 a_{2}} \frac{a_{2}\left(\nu_{\kappa}^{*}+\gamma^{* / \theta}\right)}{\nu_{\kappa}^{*}-\frac{3}{2} \zeta^{*}+3 \gamma^{*} / \theta} .
\end{gathered}
$$

Their steady values are evaluated replacing $\theta$ and $a_{2}$ by their steady values.

Figure 2 shows the time evolution of $\eta^{*}, \kappa^{*}$, and $\mu^{*}$ for $e=0.9, d=3$, and $\hat{\gamma}=0.1$. Figure 3 shows the steady values of $\eta^{*}, \kappa^{*}$, and $\mu^{*}$ as functions of $e$ for $d=3$ and $\hat{\gamma}=0.1$. As we can see from the figures, $\mu^{*}$ can be comparable with others. This situation has not been realized for free-cooling systems. 


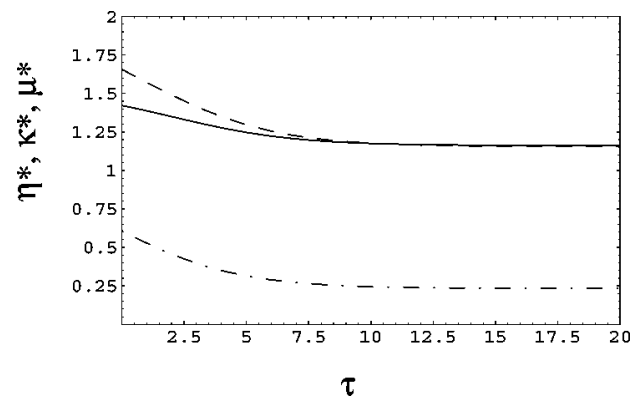

FIG. 2. The time evolution of $\eta^{*}$ (solid line), $\kappa^{*}$ (dashed line), and $\mu^{*}$ (dot-dashed line) for $e=0.9, d=3, \hat{\gamma}=0.1$, and $\theta(0)=2$.

\section{DISCUSSION AND CONCLUSION}

It is remarkable that $\mu^{*}$ is not proportional to $a_{2}$ and keeps positive for the region of $a_{2}<0$ even in the steady limit. This result is contradicted with the framework by Santos [8]. To clarify the reason, let us calculate $\partial_{t}^{(0)} \mathbf{q}^{(1)}$ in the long time limit:

$$
\begin{aligned}
\partial_{t}{ }^{(0)} \mathbf{q}^{(1)} & \rightarrow-\kappa \boldsymbol{\nabla} \partial_{t}^{(0)} T-\mu \boldsymbol{\nabla} \partial_{t}{ }^{(0)} n \\
& =-\kappa \boldsymbol{\nabla}\left[2 \gamma_{0} T_{B}-\left(2 \gamma_{0}+\zeta\right) T\right] \\
& =\kappa\left(2 \gamma_{0}+\zeta\right) \boldsymbol{\nabla} T \neq \mathbf{0} .
\end{aligned}
$$

Thus, $\partial_{t}^{(0)} \mathbf{q}^{(1)}$ cannot be zero even in the long time limit. This paradoxical situation comes from the following. In our system, the system is heated by the constant $T_{B}$ uniformly, but the temperature field $T$ contains spatial fluctuations. Thus, the mismatch of the two temperature fields survives in the long time limit. In other words, we cannot neglect the time derivative in the scaled inelastic Boltzmann equation (28) to reproduce the relaxation to the steady state.

After submission of the paper, the author has realized the existence important related papers [24-26]. In particular, the latter two papers discussed mixtures of elastic particles and granular particles. Their model reduces to our model when the density ratio and the mass ratio of two kinds of particles

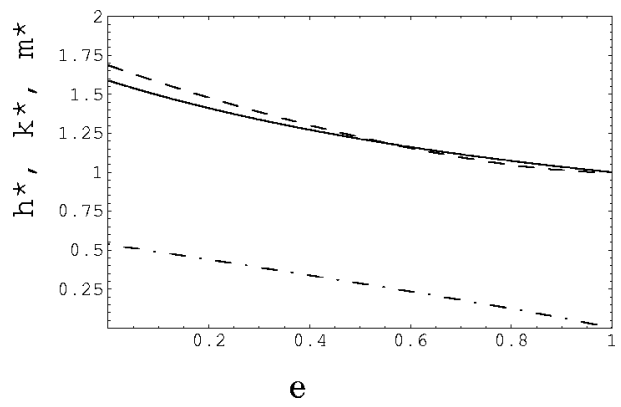

FIG. 3. The steady values of $\eta^{*}$ (solid line), $\kappa^{*}$ (dashed line), and $\mu^{*}$ (dot-dashed line) for $e=0.9, d=3$, and $\hat{\gamma}=0.1$. are large. They also include the feedback effect from granular particles to the elastic particles in the heat bath. At present, they do not discuss the spatial fluctuations but discuss only homogeneous states. Thus, the study of such a model will be fruitful. On the other hand, Garzo and Montanero [24] determined the transport coefficients in a heated system by the white noise thermostat, but their result is less related to ours, because the white noise thermostat is heated not by $T_{B}$ but by the granular temparture.

We have derived hydrodynamic equations based on a systematic Chapman-Enskog method for dilute granular gases driven by the Langevin thermostat in this paper. We have determined all the transport coefficients $\eta, \kappa$, and $\mu$ appear in Navier-Stokes order as a function of the restitution coefficient $e$.

The result is based on the linearized approximation of $a_{2}$ in the first-order truncation of Sonine expansion. Although we believe that this approximation gives a nice evaluation, nobody knows its theoretical background and quantitative validity. In particular, Pagnani et al. [10] have reported that deviation of $f$ from the Gaussian in driven granular gases is large. So we may need to check the convergence of the Sonine expansion and to compare the theoretical prediction with simulations. We also need to look for the possibility to apply our result to explain the data in actual experiments for suspensions.

\section{ACKNOWLEDGMENTS}

The author would like to thank A. Santos for fruitful discussion. This study was partially supported by the Inamori Foundation.

\section{APPENDIX A: STEADY VALUES OF $a_{2}$ AND $\theta$}

As mentioned in the text, it is possible to obtain exact steady solutions of Eqs. (25) and (29). The result is so complicated and the difference between them and the linearized solutions is small so that we do not use the exact form for later discussion. Here we present the exact solutions

$$
a_{2}^{\infty}=\frac{16\left(1-3 e^{2}+2 e^{4}\right)}{Q_{1}},
$$

where $\quad Q_{1}=73-32 e-75 e^{2}-30 e^{4}+64 d^{2} \hat{\gamma}+8 d(7+4 e$ $\left.-3 e^{2}+16 \hat{\gamma}\right)$, and

$$
\theta_{\infty}=\frac{d \hat{\gamma} Q_{1}}{Q_{2}+64 d^{3} \hat{\gamma}^{2}+Q_{3}-d(1+e) Q_{4}},
$$

where $Q_{2}=2(1+e)^{2}\left(19-46 e+33 e^{2}-12 e^{3}+6 e^{4}\right), \quad Q_{3}$ $=8 d^{2} \hat{\gamma}\left(11+4 e-7 e^{2}+16 \hat{\gamma}\right)$, and $Q_{3}=-28+e^{2}(28-30 \hat{\gamma})$ $-137 \hat{\gamma}+6 e^{3}(5 \hat{\gamma}-2)+e(12+169 \hat{\gamma})$.
[1] L. Boltzmann, Lecture Notes on Gas Theory (Dover, New York, 1995).
[2] S. Chapman and T.G. Cowling, The Mathematical Theory of Nonuniform Gases, 3rd ed. (Cambridge University Press, 
Cambridge, 1970).

[3] Granular Gases, edited by T. Pöschel and S. Luding (Springer, Berlin, 2000).

[4] S.K. Friedlander, Smoke, Dust and Haze: Fundamentals of Aerosol Behavior (Wiley, New York, 1977).

[5] T. Kawaguchi, T. Tanaka, and Y. Tsuji, Powder Technol. 96, 129 (1998); T. Kawaguchi, M. Sakamoto, T. Tanaka, and Y. Tsuji, ibid. 109, 3 (2000).

[6] K. Ichiki and H. Hayakawa, Phys. Rev. E 52, 658 (1995).

[7] J.M. Montanero and A. Santos, Granular Matter 1, 57 (1998).

[8] A. Santos, Physica A 321, 442 (2003).

[9] J.A. Carrillo, C. Cercignani, and I.M. Gamba, Phys. Rev. E 62, 7700 (2000).

[10] R. Pagnani, U.M. Bettolo Marconi, and A. Puglish, Phys. Rev. E 66, 051304 (2002).

[11] A. Goldstein and M. Shapiro, J. Fluid Mech. 282, 75 (1995).

[12] T.P.J. van Noije and M.H. Ernst, Granular Matter 1, 57 (1998).

[13] W.J. Stronge, Impact Mechanics (Cambridge University Press, Cambridge, 2000).

[14] K.L. Johnson, Contact Mechanics (Cambridge University Press, Cambridge, 1985).

[15] G. Kuwabara and K. Kono, Jpn. J. Appl. Phys., Part 1 26, 1230 (1987); N.V. Brilliantov, F. Spahn, J.-M. Hertzsch, and T.
Pöschel, Phys. Rev. E 53, 5382 (1996); W.A.M. Morgado and I. Oppenheim, ibid. 55, 1940 (1997); F. Gerl and A. Zippelius, ibid. 59, 2361 (1999); H. Hayakawa and H. Kuninaka, Chem. Eng. Sci. 57, 239 (2002).

[16] N.V. Brilliantov and T. Pöschel, Philos. Trans. R. Soc. London, Ser. A 360, 415 (2002).

[17] L. Labous, A.D. Rosato, and R.N. Dave, Phys. Rev. E 56, 5717 (1997).

[18] H. Kuninaka and H. Hayakawa, J. Phys. Soc. Jpn. 72, 1655 (2003).

[19] W.G. Hoover, Computational Statistical Mechanics (Elservier, Amsterdam, 1991).

[20] J.J. Brey, J.W. Dufty, C.S. Kim, and A. Santos, Phys. Rev. E 58, 4638 (1998).

[21] J.J. Brey and D. Cubero, Granular Gases (Ref. [3]).

[22] N.V. Brilliantov and T. Pöschel, Phys. Rev. E 61, 2809 (2000).

[23] H.-D. Kim and H. Hayakawa, J. Phys. Soc. Jpn. 72, 1904 (2003).

[24] V. Garzo and J.M. Montanero, Physica A 313, 336 (2003).

[25] T. Biben, Ph.A. Martin, and J. Piasecki, Physica A 310, 308 (2003).

[26] A. Santos, Phys. Rev. E 67, 051101 (2003). 